erythrocyte sedimentation rate, or with serum levels of antitopoisomerase I antibodies, anticentromere antibodies or C-reactive protein. Furthermore, increased serum levels of APRIL did not correlate with increased serum levels of BAFF: no patient had elevated levels of APRIL and BAFF. In comparison to the association between high APRIL levels and pulmonary fibrosis, high BAFF levels differentially associated with severe skin sclerosis.

Original article Matsushita T et al. (2007) Elevated serum APRIL levels in patients with systemic sclerosis: Distinct profiles of systemic sclerosis categorized by APRIL and BAFF. J Rheumatol 34: 2056-2062

\section{Thioredoxin might protect against oxidative-stress-induced damage in Sjögren's syndrome}

It has been suggested that oxidative stress has an important role in the pathogenesis of autoimmune diseases, including Sjögren's syndrome. Reactive oxygen species cause damage to DNA, which might lead to the generation of anti-DNA autoantibodies. The antioxidant protein thioredoxin protects against damage by reactive oxygen species, and also exhibits various immunological properties. Kurimoto and colleagues studied whether oxidative stress has a role in the pathogenesis of Sjögren's syndrome, and also whether thioredoxin has a protective effect against tissue damage caused by oxidative stress.

The study population included 29 female patients with Sjögren's syndrome, and a control group consisting of 22 patients with other rheumatic diseases (13 with rheumatoid arthritis, 9 with systemic lupus erythematosus) and 19 healthy individuals.

Higher levels of oxidative stress markers were observed in labial biopsy samples from patients with Sjögren's syndrome than from controls. Salivary levels of thioredoxin were also significantly higher in patients with Sjögren's syndrome than in controls, and high levels of thioredoxin expression were observed in acinar cells of 13 out of 19 patients. In vitro analysis showed that interferon- $\gamma$-induced IL-6 production was suppressed in thioredoxin-treated salivary gland cells, suggesting an antiinflammatory effect of thioredoxin. Furthermore, Fas-mediated apoptosis seemed to be reduced in thioredoxin-treated salivary gland cells in vitro, which suggests that thioredoxin might have an anti-apoptotic effect.

Original article Kurimoto C et al. (2007) Thioredoxin may exert a protective effect against tissue damage caused by oxidative stress in salivary glands of patients with Sjögren's syndrome. J Rheumatol 34: 2035-2043

\section{Raloxifene might be a beneficial therapy for prevention and treatment of RA}

Estrogen is thought to protect against rheumatoid arthritis (RA), since the incidence of RA peaks at menopause and use of oral contraceptives and hormone replacement therapy are known to reduce the risk of this disease. Raloxifene is a selective estrogen receptor modulator used for prevention and treatment of postmenopausal osteoporosis. It acts as an agonist of estrogen in bone but an antagonist in other tissues and, therefore, is not associated with many of the adverse effects of hormone replacement therapy. Jochems and colleagues have, therefore, studied whether raloxifene can also be used to treat arthritis.

In a well-established mouse model of RA, prophylactic and therapeutic use of raloxifene analog LY117018 resulted in reduced disease severity, joint erosion, and cartilage destruction, and a higher rate of bone formation. LY117108 treatment also delayed arthritis onset, and reduced serum levels of interleukin 6 and spleen expression of RANKL and tumor necrosis factor mRNA, when administered before the induction of disease.

The results of this study indicate that raloxifene might prove to be a beneficial therapy for prevention and treatment of RA. The authors are planning controlled clinical trials to analyze its effects in humans.

Original article Jochems C et al. (2007) Role of raloxifene as a potent inhibitor of experimental postmenopausal polyarthritis and osteoporosis. Arthritis Rheum 56: 3261-3270

\section{Does long-term bisphosphonate treatment for osteoporosis cause increased bone microdamage?}

Microdamage of bone occurs as a result of sustained fatigue loading and must be repaired, via bone remodeling, to prevent fracture. 